$\begin{array}{cl}\begin{array}{cl}\text { Revue } \\ \text { de /histoire } \\ \text { des religions }\end{array} & \text { Revue de l'histoire des religions } \\ & \begin{array}{l}4 \mid 2006 \\ \text { Varia }\end{array}\end{array}$

\title{
Karna, jeux de mots et jeux de nombres
}

Karna, on words and numbers

\section{Francine Mawet}

\section{OpenEdition}

Journals

Édition électronique

URL : http://journals.openedition.org/rhr/5208

DOI : 10.4000/rhr.5208

ISSN : 2105-2573

\section{Éditeur}

Armand Colin

\section{Édition imprimée}

Date de publication : 1 décembre 2006

Pagination : 371-387

ISBN : 978-2-2009-2106-4

ISSN : 0035-1423

\section{Référence électronique}

Francine Mawet, "Karna, jeux de mots et jeux de nombres », Revue de l'histoire des religions [En ligne], 4 | 2006, mis en ligne le 28 janvier 2010, consulté le 21 avril 2019. URL : http:// journals.openedition.org/rhr/5208; DOI : 10.4000/rhr.5208 


\section{Karna, jeux de mots et jeux de nombres}

Karna, fils de Kuntî et du Soleil et demi-frère des Pândava, est l'objet de nombreux jeux étymologiques autour de son nom, portant sur le nom de l' 'oreille », mais aussi sur ceux de la « cuirasse », du « cercle », ainsi que sur les racines "faire » et " couper », et qui soulignent le rôle particulier, presque religieux, de ce personnage complexe. Il est aussi au centre de jeux sur les nombres $(3,4,5,6,100,101)$, mettant en évidence son caractère excédentaire ou d'exclu.

\section{Karna, on words and numbers}

The name of Karna, son of Kuntî and of the Sun and half-brother of the Pândava, is therefore associated with the word for the "ear», but also with words for "cuirass », "disk» or "wheel » and with the verbs " to do » or « to cut », all this showing the particular standing, almost religious, of this complex character. On the other hand, plays on numbers $(3,4,5,6$, 100, 101) show him as in excess and excluded. 
Karna est l'un des personnages centraux, mais néanmoins assez effacé du Mahâbhârata ${ }^{1}$. Fruit de l'union du dieu solaire, Sûrya, et de Kuntî, la future épouse de Pându, il est donc le frère utérin des cinq Pândava. Abandonné à sa naissance par Kuntî et recueilli par Râdhâ, l'épouse du cocher Adhiratha, ami de Dhrtarâstra, Karna est élevé dans le clan rival des Kaurava et en devient le fidèle allié. Personnage ambigu et touchant, démesurément paradoxal, à la fois courageux et couard, généreux et respectueux des valeurs guerrières, mais aussi colérique et capable d'une profonde bassesse, il semble démentir l'opinion de Georges Dumézil, selon laquelle les héros du Mahâbhârata, contrairement à ceux de l'épopée homérique, seraient entièrement définis par leurs fonctions et ne présenteraient guère d'intérêt psychologique ${ }^{2}$. L'antagonisme particulier qui oppose, dès leur jeunesse, Karna, fils de Sûrya, et Arjuna, fils d'Indra, éclate dans le duel final, au chant 8, l'un des plus grands de toute l'épopée et des plus chargés de symbolismes ${ }^{3}$, duel au cours duquel Karna joue enfin un rôle de premier plan. Deux remarques, soulignant l'importance dévolue à ce personnage et la complexité de son rôle, porteront l'une sur les jeux étymologiques autour de son nom, l'autre sur la symbolique du nombre.

1. Peu d'études ont néanmoins été consacrées à Karna. Nous citerons principalement: Georges Dumézil, «Karna et les Pândava », Orientalia Suecana, 3 (1954), p. 60-66 ; Id., Mythe et épopée, I, Paris, 1968, p. 125-144 ; Madeleine Biardeau, Le Mahâbhârata. Un récit fondateur du brahmanisme et son interprétation, I, Paris, 2002, p. 316-323, 382-385; Alf Hiltebeitel, "Brothers, Friends, and Charioteers: Parallel Episodes in Irish and Indian Epics", Homage to Georges Dumézil, (Journal of Indo-European Studies. Monograph, $\mathrm{n}^{\circ}$ 3), Washington, 1982, p. 85-111 (part. p. 87-89 - Karnaparvan probablement bâti sur le matériel le plus archaïque du Mahâbhârata); Christophe Vielle, Le mytho-cycle hérö̈que dans l'aire indo-européenne. Correspondances et transformations helléno-aryennes, Louvain-la-Neuve, 1996, p. 147-158; John Brockington, The Sanskrit Epics, (Hdb. der Orientalistik, 2. Abt., 12. Bd.), Leyde, 1998, p. 149 ; Francine Mawet, « Le char dans l'Inde ancienne : archéologie indo-européenne et exploitation littéraire », Archiv Orientální, 73 (2005), p. 69-90 (part. p. 82-86).

2. Georges Dumézil, Mythe et épopée, I, p. 632-633.

3. Francine Mawet, Archiv Orientální, 73 (2005), p. 82-86. 


\section{LE NOM DE KARNA}

Les noms des principaux héros du Mahâbhârata ne correspondent pas nécessairement à leurs traits de caractère, leurs fonctions ou leurs liens de parenté, leurs noms ne permettent pas de déterminer automatiquement les rôles qu'ils jouent dans l'épopée ${ }^{4}$. Le nom commun karna désigne "l'oreille ». Le nom du héros se prêtait donc facilement à des jeux étymologiques, et ils sont nombreux, effectivement, mais ils portent sur bien d'autres sens encore et n'ont naturellement rien à voir avec les étymologies scientifiques modernes, qui ne peuvent en aucun cas être reportées à une époque ancienne ${ }^{5}$.

4. Georges Dumézil, Mythe et épopée, I, p. 249-250. Mais Georges-Jean Pinault, «La tradition indienne », Histoire des idées linguistiques, I. La naissance des métalangages. En Orient et en Occident, sous la direction de Sylvain Auroux, Liège-Bruxelles, 1989, p. 314-315 (à propos du Nirukta : «L'effort pour tirer des mots tout ce qu'ils peuvent signifier s'est d'abord appliqué aux noms propres, dont chacun appartient à un seul individu, et notamment aux noms des dieux, qui expriment leurs fonctions et leurs actions [...]. Ces jeux étymologiques n'hésitent pas à recourir à l'attraction paronymique, sans tenir compte des contraintes phonétiques et morphologiques. [...] D'ailleurs, des étymologies contradictoires d'un même nom sont proposées avec la même assurance »).

5. Les principales étymologies qui ont été proposées au nom de l' « oreille » sont les suivantes. Selon certains auteurs, karna-, serait rattaché à la racine *kel- "entendre" (skr. çRU-, gr. $\varkappa \lambda \varepsilon$ ćos) et reposerait sur *kol-no- (Françoise Bader, « De l'auscultation à la célérité en latin: formes de la racine *KEL- », Hommages J. Cousin. Rencontres avec l'antiquité classique, Paris, 1983, p. 2760, part. p. 30 ; id., «Principes de méthode étymologique », L'indeuropeo: prospettive e retrospettive, Milan, 1997, p. 31-80, particul. p. 64 ; pour la gutturale initiale, voir Michael Meier-Brügger, Indogermanische Sprachwissenschaft, $8^{e}$ éd., Berlin-New York, 2002, p. 129-131). Étant donné le sens «sourd » de l'adjectif védique karna- (accentué sur le suffixe, au contraire du substantif karna- « oreille »), avestique karzna- subst. " oreille», adj. " muni d'oreilles, aux longues oreilles », ainsi que « aux oreilles coupées », il a été proposé que karna- proviendrait de la réduction en une forme simple d'un ancien composé «à l'oreille coupée » (karna- $u \check{\text { - }}$ ), karna- reposant lui-même sur la racine $(s) k e r-(t)$ - « couper» : Ernst Fraenkel, MNHMH $\Sigma$ XAPIN, Gedenkschrift P. Kretschmer, I, Vienne, 1956, p. 101-104 et Litauisches etymologisches Wörterbuch, I, Heidelberg, 1962, p. 314-316. La dualité sémantique de karnaamène Manfred Mayrhofer, Kurzgefaßtes etymologisches Wörterbuch des Altindischen, I, Heidelberg, 1956, p. 172, 562-563 et Etymologisches Wörterbuch des Altindoarischen, I. Bd., L. 4, Heidelberg, 1988, p. 314-315, à retenir une racine désignant originellement «la pointe» $(* k o l-n o-$ "pointu, dirigé

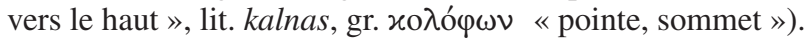


Le troisième livre du Mahâbhârata, l'Âranyakaparvan, avance une explication au nom de Karna. Ce livre traite de l'exil des Pândava pour douze ans dans la forêt. L'épisode consacré à Karna se déroule à la fin de l'exil, au début de la treizième année. Yudhisthira est préoccupé par la toute puissance de Karna, pourvu depuis sa naissance d'une cuirasse d'or et de boucles d'oreilles, dons de son père Sûrya et qui le rendent invulnérable et immortel. Indra, allié des Pândava, entreprend d'en dépouiller Karna et se présente à lui sous l'aspect d'un brâhmane mendiant, auquel Karna, lié par un voeu, ne pourra rien refuser. Entre-temps, Sûrya apparaît en songe, également sous l'aspect d'un brâhmane. Il avertit Karna des mauvaises intentions d'Indra, le dissuade de céder ses attributs et lui suggère de proposer, en contrepartie, toutes les richesses du monde, transaction que Karna refuse comme contraire à son dharma. Sûrya disparaît, intervient alors Indra. Karna n'accède pas tout de suite à sa requête, il marchande et finit par obtenir, en échange des boucles et de la cuirasse, une lance infaillible qui a pour vertu de toujours revenir dans sa main. L'accord est conclu. Mais cette lance ne pourra servir qu'une fois et contre un seul ennemi. Ici intervient la scène où Karna s'écorche lui-même à vif pour s'ôter la cuirasse : «Alors, après avoir coupé la divine cuirasse de son corps, il la donna encore ensanglantée à Vâsava. Puis, une fois coupées, il lui tendit les deux boucles d'oreilles. C'est par cet acte-là que Karna est devenu Vaikartana ». Cette dernière phrase peut aussi être traduite : «C'est par cet acte-là que Vaikartana est devenu Karna ». La question est, en réalité, secondaire, l'important étant les rapprochements sémantiques opérés entre les mots ${ }^{6}$. Cet extrait se prête à plusieurs

6. Mbh. 3, 294, 38 (éd. Poona). L'ordre des mots laisse supposer que, dans la dernière phrase, (vaikartanah karmanâ tena karnah), vaikartanah est l'apposition de karnah, mais la place du nom prédicat (attribut du sujet) n'étant pas clairement définie, un doute peut subsister dans la traduction: «Vaikartana est devenu karna » est théoriquement possible, c'est d'ailleurs la solution adoptée par Madeleine Biardeau, Le Mahâbhârata, I, p. 752. Louis Renou, Grammaire de la langue védique, Paris, 1952, § 393 («En phrase nominale, l'apposition de deux substantifs comporte normalement le prédicat avant le sujet ») ; Id., Grammaire sanscrite, $2^{\mathrm{e}}$ éd., Paris, 1968, §§ 398-399 («La place du prédicat nominal est libre ; à date ancienne, il précède le sujet, 
interprétations, qui d'ailleurs ne s'excluent pas. La première est d'établir un lien direct entre karna - «l'oreille » et le nom du héros ${ }^{7}$. Il est clair que ces boucles symbolisent quelque chose de très important chez Karna. Elles sont à la fois une marque incontestable de sa filiation avec Sûrya et un insigne royal : elles évoquent le disque ou la roue (cakra), symbole des grands rois (des cakravartin $)^{8}$. C'est d'ailleurs ce que craint par-dessus tout Yudhisthira, le roi du dharma, lui qui est appelé à régner sur terre. Il faut souligner que, dans ses mises en garde à Karna, Sûrya se montre surtout préoccupé par les boucles d'oreilles, plusieurs fois citées dans le texte, et sans référence à la cuirasse. Et dans le duel final entre Karna et Arjuna, la roue, centre dramatique de la scène, réunit un faisceau de symbolismes importants, remontant au début de la tradition indienne ${ }^{9}$. Mais Karna est également mis en rapport direct, dans cet extrait, avec l' " acte », karman $^{10}$. Karna exécute un acte extrêmement courageux en se défaisant des attributs qui lui confèrent invincibilité et immortalité, il agit en même temps selon son dharma. Rappelons par ailleurs que, dans le rituel du sacrifice en Inde ancienne, l'acte essentiel du sacrifiant est l'abandon (tyâga) de quelque chose qui lui appartient et qu'il a acquis en vue du sacrifice, en ce sens que le sacrifiant offre une partie de lui-même en abandonnant un véritable substitut de sa personne ${ }^{11}$. N'est-ce pas là justement ce que fait Karna ? Il abandonne les attributs qui le font indubitablement fils de Sûrya. Il donne plus qu'une part de lui-même, il donne sa vie, puisque, en accédant à la demande d'Indra, il signe son arrêt de mort. Cet épisode constitue un tournant décisif dans l'ensemble de l'épopée :

au moins si le sujet n'est pas un pronom ») ; Albert Thumb, Richard Hauschild, Handbuch des Sanskrit, 3e éd., Heidelberg, 1959, II, § 686 ("Das Subjekt eröffnet, das Prädikat (Verbum oder Prädikatsnomen) schließt den Satz... die Apposition kann voran- oder nachgestellt werden").

7. C'est la position adoptée, par exemple, par Christophe Vielle, Le mytho-cycle hérö̈que, p. 149.

8. Madeleine Biardeau, Mahâbhârata, I, p. 753 ; Louis Frédéric, Dictionnaire de la civilisation indienne, Paris, 1987, p. 263.

9. Francine Mawet, Archiv Orientální, 73 (2005), p. 82-86.

10. Cf. Madeleine Biardeau, Mahâbhârata, p. 441, 752.

11. Madeleine Biardeau, Charles Malamoud, Le sacrifice dans l'Inde ancienne, Paris, 1976, p. 19. 
c'est cet acte même qui rendra Karna vulnérable dans le terrible duel contre Arjuna et qui entraînera inexorablement sa mort et la victoire des Pândava sur les Kaurava. En outre, si, comme l'affirme Madeleine Biardeau, l'acte essentiel du sacrifice est l'abandon d'une partie de soi, cette partie est en quelque sorte «coupée» de soi. Karna reçoit son nom du fait qu'il commet cet «acte », l'acte du sacrifice, mais aussi parce qu'il « coupe » ou « découpe » son corps, comme on découpait les victimes destinées au sacrifice. C'est ce que suggèrent les rapprochements, dans ce passage, entre Karna et des formes de la racine $K \mathrm{R} T$ - « couper », une telle insistance dans les termes évoquant naturellement plus qu'une simple égratignure sur le corps de Karna (quatre formes du verbe $K \mathrm{R} T$ - « couper » en quatre çloka: Mbh. 3, 294, 35 akrntata, 36 nikrntantam, 37 samkrttagâtram, 38 utkrtya, ainsi que le synonyme chittvâ «ayant découpé » 38), tout comme le nom Vaikartana, constitué par vrddhi, sur l'épithète de Sûrya Vikartana "Sûrya le diviseur ${ }^{12}$. Après un tel acte de mutilation et cinq allusions claires à celui-ci, dans le même passage, à travers la racine $K R T$ - « couper », il serait naturel que Karna soit perçu comme «le découpé » ou «l'écorché ». On peut, en dernier lieu, se demander si la mutilation évoquée concerne l'opération de découpage de la cuirasse ou s'il est fait plus précisément allusion au don des boucles d'oreilles, évoqué immédiatement avant. Dans ce cas, Karna évoquerait un emploi ancien, védique du mot, comme adjectif, «muni d'oreilles, aux longues oreilles », mais aussi « aux oreilles coupées », sens que partage d'ailleurs aussi l'avestique ${ }^{13}$, et qui n'était peut-être pas complètement sorti du sentiment linguistique, les poètes ayant sans doute joué un rôle important dans l'établissement de connexions sémantiques entre diverses racines autour du nom de Karna. Ou, au contraire (et peut-être en même temps), c'est la cuirasse, carman-, héritée de son père (et la mutilation qui en découle), qui

12. Sûrya a reçu le nom de Vikartana «le diviseur », parce que, dit-on, il s'est divisé en deux parties, l'une étant restée au ciel et l'autre étant descendue sur terre pour s'unir à Kuntî et poursuivre sa vie en Karna : Christophe Vielle, Le mytho-cycle hérö̈que, p. 150, n. 256.

13. Christian Bartholomae, Altiranisches Wörterbuch, Strasbourg, 1904 (reprint Berlin-New York, 1979), col. 455 (kardna- «taub») et $c f$. l'étymologie du mot n. 5 
caractérise ici le héros. Le nom de la cuirasse est également apparenté à la racine $* k r(t)$ - « couper ${ }^{14}$, ce qui était peut-être aussi perçu des poètes épiques ${ }^{15}$. En outre, la mythologie indo-européenne nous livre deux autres faits intéressants. Il s'agit premièrement d'un parallélisme entre Karna et le héros grec Memnon, mis en évidence par Christophe Vielle ${ }^{16}$, plusieurs éléments précis unissant les deux héros. Le plus interpelant, en ce qui nous concerne, c'est que chacun des deux guerriers est doté d'une cuirasse de nature divine et invulnérable. L'autre point de comparaison est un récit épique ossète, dans la légende des Nartes, étudié par Georges Dumézil ${ }^{17}$, dont le thème central est la cotte de mailles du soleil. Quoique le contexte en soit bien distinct de celui du Mahâbhârata, les rapprochements avec le sort de Karna sont assez surprenants. Que ce soit la cotte de mailles ou la cuirasse de Karna, toutes les deux sont un présent du soleil : d'un côté, chez les Ossètes, le soleil offre sa propre cotte de mailles à sa fille, qui doit elle-même la remettre à son beau-père humain, Uærxtænæg, de l'autre, il fait naître Karna avec une cuirasse dorée et infaillible, en tous points identique à la sienne. Jaloux, les ennemis des Nartes attaquent Uærxtænæg, lui ôtent sa cotte de mailles et découpent deux bandes de peau sur son dos. Cette opération fait curieusement penser à la dissection opérée par Karna. Un fois remises en place, ces bandes se fondent dans la peau sans laisser de trace, de la même manière que Karna a obtenu d'Indra que son corps ne garde aucune trace de son autotomie. Selon Georges Dumézil, cet épisode appartiendrait à une ancienne tradition épique, dont il n'existe même aucune allusion védique.

Il est important de souligner que toutes les connexions sémantiques sont issues de ce même épisode du Mahâbhârata, celui où Karna se dépouille lui-même des attributs qui le rendaient à la fois immortel et unique en son genre, et ceci n'est sans doute pas fortuit.

14. Manfred Mayrhofer, KEW, I, p. 378.

15. L'alternance $k / c$ étant encore vivante dans la langue : Louis Renou, Grammaire sanskrite, § 45-46.

16. Christophe Vielle, Le mytho-cycle hérö̈que, p. 147-158.

17. Georges Dumézil, Mythe et épopée, I, p. 53-102 ; Christophe Vielle, Le mytho-cycle hérö̈que, p. 193-194. 


\section{JEUX DE NOMBRES}

Les nombres revêtent une grande importance tout au long du récit du Mahâbhârata. La biographie de Karna, particulièrement, nous livre cinq nombres significatifs $(3,4,5,100$ et 101$)$. Si, d'après la place qu'il occupe au sein de sa famille, Karna se rattache au système trifonctionnel analysé par Georges Dumézil ${ }^{18}$, il semble cependant absent de ce schéma. Il présente, en effet, bien des affinités avec le caractère et les aptitudes de son demi-frère Arjuna qui le rapprocheraient de la deuxième fonction, qu'il occupe d'ailleurs de fait en tant que roi d'Anga. Néanmoins, on ne sent pas qu'il soit vraiment défini par les traits spécifiques de cette fonction. D’ailleurs rien dans les faits ni les personnes qui l'entourent ne semble l'implanter dans le schéma trifonctionnel.

À travers ses demi-frères, les Pândava, Karna s'inscrit plus clairement dans la symbolique du nombre cinq. Ce chiffre délimite un ensemble de choses qu'on ne peut diviser, un ensemble auquel on ne peut rien enlever ni ajouter, selon un symbolisme bien attesté dans les traditions indo-iraniennes et indo-européennes en général ${ }^{19}$. Le nombre des Pândava en est un bel exemple et il serait difficile de

18. Par exemple, dans Mythe et épopée, I, p. 81-130, mais aussi, en remontant dans l'arbre généalogique, l'analyse de Daniel Dubuisson, « La préhistoire des Pândava », Revue de l'Histoire des Religions, 202, 1985, p. 227-241.

19. Edgard C. Polomé, "The Indo-European Numeral for 'five' and Hittite panku- 'all'”, Pratidânam Kuiper, La Haye, 1969, p. 98-101 ; Nadia Van Brock, « De $\pi v \dot{\xi}$ à $\pi \tilde{\alpha} \varsigma$ », Mélanges de linguistique et de philologie grecques offerts à Pierre Chantraine, Paris, 1972, p. 263-276 ; Francine Mawet, « Cinq et sept en iranien ancien », Humour, travail et science en Orient, Louvain, 1988, p. 287-304 (et bibl. n. 49) ; Winfred P. Lehmann, "Linguistic Structure as Diacritic Evidence on Proto-Culture", Indo-Europeans and Indo-European, Philadelphie, 1970, § 3.9 ; Louis Renou, Jean Filliozat et al., L'Inde classique. Manuel des études indiennes, I, Paris, 1985, 684 ; Abel Bergaigne, La religion védique d'après les Hymnes du Rig-veda, 2e tirage, Paris, 1963 (= 1 ${ }^{\mathrm{re}}$ éd. 1878-1883), II, p. 114-129. Jeux sur le nombre cinq particulièrement dans la Brhad-âranyaka-upanisad, Emile Senart, Paris, 1967 (analyse, p. X-XXVII) ; Aliette Silburn, çvetâçvatara Upanisad, Paris, 1978, § 19, p. 27 sqq., § 33, p. 44 ss.; M. Johnston, Early Sâmkhya, 1937, p. 16-21 ; Anne-Marie Esnoul, L'hindouisme. Textes et traditions sacrés, Paris, 1972, not. p. 90, 96-99. Cf. Chândogya-up., 3.13 (éd. Emile Senart, Paris, 1971). 
croire qu'il soit un pur produit du hasard ou un choix totalement arbitraire des poètes. On sait que, lors du mariage (svayamvara) de Draupadî, une épreuve de tir à l'arc fut organisée pour départager les prétendants de la jeune fille. Aucun n'était arrivé à bander la corde de l'arc jusqu'à l'arrivée de Karna. Mais, alors qu'il est prêt de réaliser l'exploit, celui-ci, humilié, est exclu de l'épreuve par Draupadî, en tant que « fils de cocher» $(M b h .1 .178)$. Arjuna remportera l'épreuve et, suite à une discussion entre eux, Draupadî deviendra l'épouse commune des cinq Pândava. Le chiffre cinq est central dans ce dernier épisode : Arjuna envoie d'un seul coup cinq flèches dans la cible et Draupadî est désignée comme la femme de cinq époux. Selon Madeleine Biardeau, ce chiffre a une connotation particulière : les cinq époux forment un ensemble indivisible et sont à mettre en relation avec les cinq feux sacrificiels de l'hindouisme ${ }^{20}$. Les cinq feux du sacrifice solennel sont composés des trois feux principaux auxquels s'ajoutent deux feux secondaires, ce qui correspond à la manière dont se scinde le groupe des Pândava, les deux cadets ayant été enfantés par Madrî, une vaiçyâ. Les cinq sacrifices seraient une version des trois dettes congénitales que contracte tout homme à la naissance, et qui sont montées ici à cinq, les trois dettes originelles que sont les sacrifices rendus aux dieux, au brahman et aux ancêtres, et les deux dettes secondaires que sont les sacrifices aux hommes et aux créatures inférieures ${ }^{21}$. La symbolique du nombre cinq qui entoure les rites sacrificiels pourrait donc être mise en parallèle avec celle des cinq Pândava. Un autre élément corrobore cette interprétation. Draupadî est surnommée Yajñasenî, surnom qui lui vient de son père Drupada, également appelé Yajñasena et roi des Pañcâla. Ce nom lie directement Drupada au sacrifice (yajña) qu'il a célébré dans l'espoir d'obtenir un fils. Or, yajñasena sert à désigner un arbre, mais surtout le poteau sacrificiel. L'image que reflète le quintuple mariage de Draupadî pourrait donc être celle des cinq sacrifices

20. Madeleine Biardeau, Mahâbhârata, I, p. 280-284 ; Madeleine Biardeau, Charles Malamoud, Le sacrifice dans l'Inde ancienne, p. 40 ; Abel Bergaigne, La religion védique, II, p. 149 (cf. çatapatha-Brâhmana, 11. 5. 6. 1-3, trad. Julius Eggeling, V, Delhi-Bénarès, 1972 [= 1900], p. 95-96).

21. Madeleine Biardeau, Charles Malamoud, Le sacrifice, p. 38-41. 
indissociables, disposés autour du poteau sacrificiel. Dans ce contexte, il est naturel que les Pândava soient cinq et que les frères partagent une seule épouse ou que Draupadî soit unie à cinq maris exactement ${ }^{22}$. Cette sorte de totalité représentée par le chiffre cinq et le rapport étroit qu'il entretient avec le sacrifice sont clairement explicités dans d'autres textes védiques, par exemple dans la Brhad-âranyakaupanisad (BÂU, 1, 4, 17) ${ }^{23}$. Les cinq Pândava forment un groupe uni. Karna, même s'il en est issu, est exclu de ce groupe. Plusieurs épisodes marquants de sa vie le prouvent, le plus significatif étant sans nul doute celui de sa naissance. Dès les premiers instants de sa vie, Karna est écarté de la branche des Pândava, de la caste des $k$ satriya. Recueilli par une famille de cochers, il portera douloureusement le surnom de sûtaputra «fils de cocher». Karna est une seconde fois évincé dans sa jeunesse lors de sa rencontre avec le brâhmane Râma Jâmadagnya ( $M b h .12,2-3)$, auprès de qui il va parfaire sa formation en sciences des armes, en se faisant passer lui-même pour un brâhmane. Mais il commet deux erreurs qui lui seront fatales et lui vaudront deux malédictions. En premier lieu, alors qu'il se promenait sur le rivage de l'océan, il tue en toute ignorance le veau de la vache à oblations d'un brâhmane très pieux. Karna implore le pardon du brâhmane, ce que celui-ci lui refuse. Il lui prédit que, lors d'un combat, la terre avalera la roue de son char et que son ennemi lui tranchera la tête, ce qui se réalisera effectivement. Une deuxième malédiction le frappe lorsqu'un jour Jâmadagnya s'endort, la tête posée sur ses genoux. Un ver commence à percer la jambe de Karna, à lui dévorer l'intérieur pour en sucer le sang.

22. Madeleine Biardeau, Mahâbhârata, I, p. 282-283; Jean-Michel Péterfalvi, Madeleine Biardeau, Le Mahâbhârata, I, Paris, 1085, p. 117 (Pañcâla formé sur pañca « cinq »).

23. $B \hat{A} U, 1.4 .17$ (trad. Emile Senart, Paris, 1967) (digression sur l'âtman en relation avec le sacrifice): "Aussi longtemps que lui manque un seul de ces objets, il se sent incomplet. Voici sa plénitude : la pensée est son âtman, la parole son épouse, le souffle (la vie) sa progéniture, sa vue les biens terrestres - car c'est seulement par la vue qu'on les découvre -, l'ouïe les biens célestes - car c'est par l'ouïe que se perçoit l'enseignement; l'âtman même est son activité, car c'est par l'âtman qu'on agit. Quintuple est le sacrifice, quintuple la victime, quintuple l'homme ; tout ce qui existe est quintuple. Il obtient tout ce qui existe celui qui sait ainsi. »Cf. aussi, par exemple, Chândogya-upanisad, 2, 21. 
Malgré la douleur atroce, Karna ne bronche pas de crainte de réveiller son maître assoupi. Celui-ci découvre cependant avec horreur la chair ensanglantée de son disciple et l'accuse de ne pas être un brâhmane, car seuls les ksatriya sont capables de supporter de telles souffrances. Karna dévoile alors son identité et, pour avoir osé lui mentir par convoitise des armes, Jâmadagnya prédit à son tour à Karna qu'il oubliera la science du brahmâstra face à son ennemi. À la suite de ces deux événements, Karna est mis en dehors du cercle des brâhmanes, la connaissance des armes lui sera ôtée au moment où elle lui sera indispensable. Surtout l'heure et les conditions de sa mort lui sont prédites. Plus tard, lorsque Draupadî refuse à Karna le droit de participer à l'épreuve du svayamvara, il se trouve rejeté du cercle des Pândava, des ksatriya, et, dans le même temps, du cercle sacrificiel dans lequel sont implantés ses cinq demi-frères en s'unissant à Draupadî. L'ultime répudiation que doit subir Karna est évidemment son exécution par Arjuna sur le champ de bataille, conformément aux prédictions qui lui avaient été faites par les deux brâhmanes. Une dernière fois, le sixième frère est mis au ban du groupe, il est le seul des Pândava à perdre la vie au cours de la guerre. Dans un tel cadre, la biographie de Karna donne réellement l'impression qu'il est la personne «en trop » au sein des Pândava et qu'il doit être éliminé. Avant cet épisode ultime, Kuntî essaie en vain, après un échec de Krsna d'ailleurs, de convaincre Karna de quitter le clan des Kaurava pour se rallier à celui des Pândava, et ainsi gagner une chance de survie. Mais Karna refuse, par fidélité aux Kaurava qui l'ont soutenu depuis le début, par rancune aussi vis-à-vis de sa mère qui l'a délaissé à sa naissance. Néanmoins, avant le dernier duel, il fait à sa mère une promesse solennelle et lourde d'émotion : $M b h$. 5. 144, 20-22 «Mais cette initiative que tu as prise auprès de moi ne sera pas vaine. À l'exception d'Arjuna, je ne tuerai pas tes fils, Yudhisthira, Bhîma et les jumeaux, même si je suis capable de leur résister au combat et qu'ils méritent la mort. Parmi les guerriers de Yudhisthira, je ne combattrai qu'avec Arjuna, égal à moi. Ou bien je suis rempli de mérite en le tuant au combat, ou bien je suis couvert de gloire si je suis tué par Savyasâcin (Arjuna). Tes cinq fils ne périront pas, ô femme glorieuse, soit tu resteras avec Karna sans 
Arjuna, soit avec Arjuna si je suis tué ». Cette dernière phrase, d'une remarquable concision, ne peut être plus claire. Ce que promet Karna à sa mère corrobore l'idée que les Pândava doivent rester cinq quoiqu'il advienne. Kuntî aura toujours cinq fils, pas un de plus, pas un de moins. Karna participe à cette logique numérique, il assure au groupe son chiffre originel et n'ébranlera pas cette sorte d'ordre fondamental des choses que semblent représenter les cinq Pândava. Tous ces faits confirment que le nombre cinq détient une signification particulière à l'intérieur de l'épopée. Il en ressort une notion de globalité, d'ensemble, de totalité. La récurrence de références au nombre cinq et le rapport de ce nombre avec les rites sacrificiels ne sont pas dus au hasard, mais ont un sens. Les Pândava représentent effectivement, dans le Mahâbhârata, le camp gagnant, celui du dharma. C'est eux qui éliminent les puissances susceptibles de nuire à l'ordre du monde et se chargent ensuite de le maintenir. C'est pour ces raisons qu'ils sont liés au « Tout » et au sacrifice, garant de l'ordre des mondes.

Ajoutons à cette analyse un autre épisode de la vie de Karna (Mbh. 1. 127). Il s'agit du concours de tir à l'arc organisé par leur tuteur Drona entre les jeunes princes, Kaurava et Pândava, au terme de leur éducation. À la fin du spectacle, Karna surgit et provoque Arjuna en duel. Mais l'épreuve avorta. Indra envoie des nuages d'orage pour protéger son fils Arjuna, tandis que Sûrya entoure Karna de rayons lumineux. Il est alors demandé à Karna de quelle lignée royale il est issu, Arjuna ne pouvant se battre contre un adversaire autre que ksatriya. Connaissant les origines de Karna, Duryodhana intervient in extremis et le fait sur le champ roi d'Anga. Karna est donc ksatriya. Malheureusement, l'arrivée inopinée de son père adoptif Adhiratha, auquel il rend hommage comme il se doit, l'exclut définitivement et honteusement de l'épreuve. Bhîma s'écrie que son statut de sûtaputra le rend indigne de régner sur le royaume d'Anga et qu'il devra mourir des mains d'Arjuna. À ces mots, Karna jette un regard de désolation vers le soleil, son père. À propos de cette anecdote, on peut se demander pourquoi Karna est nommé roi d'Anga. Il est curieux de souligner qu'outre le nom du peuple, anga désigne un «membre du corps » ou le «corps » en 
entier. Anga correspond aussi au nombre six, aux six membres du corps (les bras, les jambes, le tronc, la tête) ${ }^{24}$. À travers ce titre de roi d'Anga, il y a donc peut-être aussi une allusion au fait que Karna devrait être considéré comme partie intégrante du groupe des Pândava, en tant qu'élément d'un corps entier.

Si Karna est le frère excédentaire des cinq Pândava, il est aussi l'aîné véritable des quatre fils de Kuntî et, en ce sens, il figure une fois de plus comme un personnage à part, qui, dans un sens, a un lien avec le groupe, mais, dans un autre, s'en distingue, le nombre quatre se présentant, dans la pensée indienne, comme l'addition de trois plus un ${ }^{25}$. Dans ce cadre, également, il est le frère excédentaire par excellence, un élément qui se détache de l'une ou l'autre manière d'un groupe bien défini, d'un ensemble complet et parfait.

Il serait intéressant de se demander si Karna est bien le seul à occuper cette situation de « fils de trop ». Il est un personnage dont on parle peu dans le Mahâbhârata. Il s'agit de Yuyutsu. Peu de passages le concernent personnellement, c'est une figure secondaire, effacée. Cependant certains extraits ne sont pas sans intérêt, en parallèle avec la figure de Karna. Il s'agit particulièrement du passage décrivant les conditions dans lesquelles il est né (Mbh. 1. 107, 34-37). Yuyutsu est le fils que Dhrtarâstra a eu d'une vaiçyâ, alors que Gândhârî en était à un stade avancé de la gestation des cent Kaurava. Dhrtarâstra a cent fils et une fille enfantés par Gândhârî, et un cent unième fils issu de l'union avec une femme de rang inférieur. Yuyutsu occupe d'une certaine manière une place similaire à celle de Karna par rapport à ses frères, le schéma étant ici « $100+1$ ». Il est qualifié de karana, du fait qu'il est né d'un ksatriya et d'une vaiçyâ. Pour ce

24. Abel Bergaigne, La religion védique, II, p. 121-122 ; Georges Ifrah, Histoire universelle des chiffres, Paris, 1981, p. 472.

25. Anne-Marie Esnoul, « À propos de catur», Studia Iranica, 11 (1982), p. 83-88. Anne-Marie Esnoul cite, par exemple, la «triple science », les trois plus anciennes samhitâ védiques, auxquelles a été ajouté l'Atharvaveda, les quatre sortes d'officiants, parmi lesquels le brâhmane est le seul à ne rien dire, les quatre castes, dont seules les trois premières relèvent du schéma trifonctionnel indo-européen, les quatre étapes de la vie du brâhmane, dont seules les trois premières sont liées au sacrifice quotidien et à l'entretien du feu. Abel Bergaigne, La religion védique, II, p. 124-126. 
qui touche à sa naissance, il se présente en quelque sorte comme l'inverse de Karna, puisqu'il est lié aux Kaurava par son père et issu d'une caste inférieure par sa mère, tandis que Karna est Pândava par sa mère et «fils de sûta» par ses parents adoptifs. Mais tous deux ont une origine et une naissance particulières par rapport à leurs propres frères et font figure d'élément unique, débordant d'un ensemble défini de personnes. Qui plus est, au moment de la guerre, Yuyutsu, comme Karna, combat pour le camp opposé à celui de sa famille d'origine. Cependant, contrairement à Karna, Yuyutsu n'a pas été abandonné, il a toujours vécu entouré des Kaurava et n'a apparemment aucune raison de leur en vouloir. Mais une attitude de Yuyutsu nous laisse deviner qu'il a choisi son camp, et cela bien avant que ne se produisent les événements qui conduiront au conflit entre les deux familles. Juste après les funérailles de Pându et de Madrî, les Pândava et les Kaurava, encore jeunes, réunis sous la garde de Dhrtarâstra, laissent poindre la haine qui les opposera plus tard. Les Pândava se montrent plus puissants que les Kaurava aux jeux de combat et déjà Duryodhana jalouse la force sans mesure de Bhîma. À deux reprises, il tente d'empoisonner Bhîma. Lors de la deuxième tentative, Yuyutsu, "par souci du bien-être des cinq Pândava », les en avertit ${ }^{26}$. Ceci donne un indice du penchant de Yuyutsu pour les Pândava, tendance qui ne fera que se confirmer. Au début des combats, Yudhisthira, à la surprise générale, se défait de ses armes et, accompagné des autres Pândava et de Krsna, s'approche de Bhîsma, Drona, Krpa et çalya, implore leur bénédiction et demande la permission de lutter contre eux. Krsna s'avance ensuite vers Karna et Yuyutsu. Il propose à Karna de combattre dans le clan des Pândava jusqu'à la mort de Bhîsma, qu'il hait, ce que Karna refuse. Mais lorsque Yudhisthira appelle les autres soldats à se joindre à eux, Yuyutsu « posa alors son regard sur eux et, le coeur heureux, il adressa ces mots à [...] Yudhisthira [...] : "Moi, je combattrai pour votre cause contre les fils de Dhrtarâstra, ô grand roi, si tu m'acceptes, toi qui es sans péché" ${ }^{27}$. Voilà comment Yuyutsu se désolidarise de ses frères en s'engageant du côté des Pândava. À ce

26. Mbh. 1. 119, 39-40.

27. Mbh. 6. 41, 90-91. 
moment précis du récit, Karna et Yuyutsu se démarquent en prenant deux voies radicalement opposées. Cet antagonisme est d'autant plus marqué que le texte les fait intervenir directement l'un après l'autre et que Yuyutsu est le seul des Kaurava à se manifester. Les Pândava sont alors quasiment assurés de leur victoire. C'est comme si Yuyutsu en avait le présage devant les yeux, il saute sur l'unique occasion de passer dans le camp des futurs vainqueurs avant le massacre. Yudhisthira déclare d'ailleurs à Yuyutsu qu'il est déjà désigné pour organiser les funérailles des fils de Dhrtarâstra. Vu sous un autre angle cependant, Yuyutsu est le seul à abandonner les Kaurava, il est le cent unième des fils de Dhrtarâstra et se détache d'un groupe de cent fils, comme Karna le fait des cinq Pândava. Nous touchons ici au symbolisme du nombre 101, qui rejoint les mêmes valeurs que le nombre cinq. À ce propos, nous évoquerions d'abord un extrait de la Chândogya-upanisad $(8,6,6)^{28}$ «Il y a cent une veines au cœur; dans le nombre une seule s'élève vers la tête ; celui qui monte par celle-ci va à l'immortalité ; par les autres on sort dans toutes les directions - on sort dans toutes les directions ». Il est surprenant que, dans cet extrait, une seule veine parmi les cent une se dirige vers la tête et atteint l'immortalité. De même Yuyutsu, choisissant le clan des Pândava, choisit le camp de la vie, du dharma, donc de l'immortalité. De plus, un mot de la stance confirme l'idée que ceux qui restent sont voués à la mort. En effet, utkramana- nt. « le fait de sortir » (formé sur le verbe $u t-K R A M$ - « sortir, s'échapper », mais aussi « mourir ») peut également désigner « la mort » ${ }^{29}$. Ce qui, dans l'épisode du Mahâbhârata, est la situation des demi-frères de Yuyutsu destinés à mourir. Le nombre 101, comme le nombre 5, est, en outre, associé au sacrifice dans RV. 10. 130. 1 ( « Le sacrifice qui de toutes parts est tendu avec des fils, qui s'étire sur cent un actes divins » $)^{30}$. Dans cet extrait, contrairement à l'extrait précédent, cependant, l'unité qui déborde la centaine n'est pas tant un élément

28. ChU. 8. 6. 6 (trad. Emile Senart, Paris, 1971).

29. Sir Monier Monier-Williams, A Sanskrit-English Dictionary, $n^{\text {elle }}$ éd., Oxford, 1979 (= 1899), p. 177.

30. RV. 10. 130. 1. Trad. Louis Renou, Les hymnes spéculatifs du Véda, Paris, 1956, p. 127. 
distinct et opposé aux cent autres, mais plutôt un élément « stabilisateur », une sorte de limite à l'infinitude du nombre cent. Le Rgveda mentionne plusieurs fois le nombre cent et, en général, il sert à marquer une grande quantité. Les exemples sont nombreux. Nous retiendrons particulièrement $R V$. 2. 18. 6 (hymne à Indra) ( [Indra] convoyé par cent alezans ») qui se situe au terme d'une énumération de 4, 6, 8, 10, 20, 30, etc., 90 alezans ( $R V .2$. 18. 4-6), pour culminer à la strophe 7 avec «tous les alezans ${ }^{31}$. Le cent unième élément apparaît cependant aussi comme élément « stabilisateur ». Ainsi, dans l'hymne RV. 1. 117, 17-1832, Rjrâçva, qui a perdu la vue suite à une malédiction, immole d'abord cent béliers, puis un cent unième, sacrifice grâce auquel les Açvin lui rendent la vue. On peut penser que ce passage de cent à cent un permet à Rjrâçva d'accomplir un sacrifice complet. Les cent un béliers évoquent les cent (un) Kaurava, d'autant que la cécité de Rjrâçva évoque celle qui afflige Dhrtarâstra depuis sa naissance, cécité caractéristique, rappelons-le, des sages dans la tradition védique et indo-européenne en général ${ }^{33}$. Mais, contrairement aux cent un béliers sacrifiés dans l'hymne védique, Yuyutsu est l'unique survivant des Kaurava, les cent autres ayant été sacrifiés pour le rétablissement du dharma. Un court épisode du Mahâbhârata fait allusion à ce sacrifice des cent Kaurava. Lorsque Dhrtarâstra exprime publiquement le voeu de se

31. Louis Renou, Études védiques et pâninéennes $(=E V P)$, XVII, Paris, 1969, p. 67. Voir aussi les cent chars d'Usas (RV. 1. 48.7 « celle-ci se rend chez les hommes avec cent chars »-commentaire de Louis Renou, EVP, III, Paris, 1957, p. 22, «multiplication interne par projection des U. prises ensemble »), cent vaches $(R V .5 .61 .10)$ ou répétition de çatam « cent » pour exprimer la durée de vie (RV. 10. 161. 3-4), etc. Remarque de A. Langlois, Rig-véda ou livre des hymnes, $2^{\mathrm{e}}$ éd. Ph.-Ed. Foucaux, Paris, 1872 (= 1964), p. 113b, n. 5.

32. Abel Bergaigne, La religion védique, III, p. 6, 10. RV. 1. 117. 17 (çatam mesân), 18 (çatam ekamca mesân).

33. Georges Dumézil, Mitra-Varuna. Essai sur deux représentations indoeuropéennes de la souveraineté, 3e éd., Paris, 1948, ch. IX, p. 163-187 ; id., Mythe et épopée, I, p. 157 ; Jan Gonda, The Vision of the Vedic Poets, La Haye, 1963 ; Françoise Bader, «Introduction à l'étude des mythes indo-européens de la vision. Les Cyclopes », Studi Indo-Europei, éd. Enrico Campanile, Pise, 1985, p. 9-50 ; id., La langue des dieux, ou l'hermétisme des poètes indoeuropéens, Pise, 1989, ch. I, part. § 5, 10-11 ; id., L'indeuropeo: prospettive e retrospettive, $\S 8$. 
retirer dans la forêt pour mener une vie d'ascète, il se rend chez Vyâsa pour être initié aux règles de la vie érémétique. Il est ensuite recueilli dans l'ermitage du rsi dont le nom çatayūpa «aux cent poteaux sacrificiels » renvoie clairement aux victimes sacrificielles de la guerre qu'ont été les cent fils de Dhrtarâstra. Çatayûpa est, pour le vieux roi, le souvenir vivant des cent fils disparus par sa propre faute ${ }^{34}$. Les brâhmanes lui avaient, en effet, conseillé de se débarrasser de Duryodhana à sa naissance, mais, pris d'affection pour son fils, il n'avait pas suivi ce conseil, et avait fait de Duryodhana le chef des Kaurava. Au début de sa vie en ermitage, les Pândava, Vyâsa et d'autres rsi viennent lui rendre visite. Vyâsa propose d'accorder une faveur à chacun des convives. Dhrtarâstra souhaite ne plus souffrir en pensant à ses fils et à la mauvaise nature de Duryodhana, attisant la douleur des mères et épouses des guerriers morts. Entendant cela, Vyâsa leur promet de les revoir tous. Il plonge dans la rivière et rappelle les guerriers des deux camps, tués sur le Kuruksetra, et «Dhrtarâstra, les voyant tous par une vision divine, (...) se remplit de joie grâce à la faveur de l'ascète » $(M b h .15 .40,21)$. Grâce à une vision divine que lui a accordée Vyâsa, le vieux roi voit ses fils pour la première fois de sa vie ${ }^{35}$.

La figure complexe de Karna se trouve ainsi au centre d'un réseau de symbolismes, très nombreux, qui culminent dans le grand duel final et compensent quelque peu l'ombre dans lequel il est généralement laissé, lui l'exclu, le rejeté par excellence ${ }^{36}$.

Département de Langues et Littératures anciennes

Faculté de Philosophie et Lettres - CP 175

Université Libre de Bruxelles

50 av. Fr. Roosevelt

B 1050 - Bruxelles

34. Madeleine Biardeau, Mahâbhârata, t. II, p. 673.

35. Vision retrouvée qui, d'après l'analyse de Georges Dumézil, serait aussi la reconnaissance de la fatalité qui l'a entraîné dans cette guerre, Dhrtarâstra ayant, en quelque sorte, une nouvelle « vision» des terribles événements qui viennent de se passer, puisque, effectivement, selon lui, les héros de l'épopée seraient entièrement déterminés par leur destin : Georges, Dumézil, Mythe et épopée, I, p. 162-170.

36. Cet article a pour point de départ un travail réalisé sous notre direction par Mathieu Danero Iglesias en 2003. 
